Europhysics Letters

PREPRINT

\title{
Synchronization and frustration in oscillator networks with attractive and repulsive interactions
}

\author{
DAMiÁN H. ZANETte \\ Consejo Nacional de Investigaciones Científicas y Técnicas, Centro Atómico Bariloche \\ and Instituto Balseiro, 8400 Bariloche, Río Negro, Argentina \\ PACS. 05.65.+b - Self-organized systems. \\ PACS. 05.45.Xt - Synchronization; coupled oscillators.
}

\begin{abstract}
We study, numerically and analytically, the stability of synchronization for an ensemble of coupled phase oscillators with attractive and repulsive interactions, as a function of the number of repulsive couplings and their intensity. Scaling properties of the desynchronization transition are disclosed, and it is shown that unsynchronized configurations have different symmetries depending on the intensity of the repulsive interaction. The concept of frustration minimization helps understanding the main features observed in the collective dynamics of the oscillator network.
\end{abstract}

Synchronization is a well-known form of collective dynamics in large ensembles of interacting dynamical systems. Under the action of attractive coupling, strong correlations develop in time between the states of individual elements, and the ensemble is able to generate signals of macroscopic amplitude. This coherent behaviour is found in a broad class of natural systems -especially, in the realm of life sciences [1]- and has been reproduced by means of a variety of mathematical models [2-4].

The strongest manifestation of synchronized dynamics, which can be realized in an ensemble of identical periodic oscillators subject to attractive coupling, is full synchronization. In a fully synchronized ensemble, the individual motions of all the oscillators coincide. Heterogeneities, chaotic individual dynamics, and repulsive interaction, on the other hand, may inhibit the development of synchronization [4].

In this Letter, we explore the competing effect of attractive and repulsive interaction in oscillator ensembles. Specifically, we study the influence of repulsive couplings on the stability of full synchronization. The combined action of attractive and repulsive couplings has already been considered by Daido [5-7], who disclosed the possibility of weaker forms of synchronized dynamics, glass-like behaviour, and anomalous relaxation. These results, however, typically apply to cases where the number of attractive and repulsive couplings and their relative intensity are equal on the average. Here, instead, we are interested at the effect of repulsive couplings as their abundance and intensity are varied gradually. As discussed below, this leads naturally to the consideration of heterogeneous random networks of coupled oscillators.

We consider an ensemble of $N$ identical oscillators, whose individual states are given by the phases $\phi_{i} \in[0,2 \pi)(i=1, \ldots, N)$. In the absence of interaction, the evolution of the

(C) EDP Sciences 
phases is given by $\dot{\phi}=\omega$ for all $i$. Coupling is introduced following Kuramoto's scheme [2],

$$
\dot{\phi}_{i}=\omega+\frac{1}{N} \sum_{j=1}^{N} W_{i j} \sin \left(\phi_{j}-\phi_{i}\right)
$$

where $W_{i j}$ weights the interaction between oscillators $i$ and $j$. By transforming $\phi_{i} \rightarrow \phi_{i}+\omega t$ for all $i$, we fix, without generality loss, $\omega=0$. In the following, we study the case of symmetric coupling, $W_{i j}=W_{j i}$. For each realization of the ensemble, the interaction weights are chosen at random as

$$
W_{i j}=\left\{\begin{aligned}
1 & \text { with probability } 1-p \\
-w & \text { with probability } p
\end{aligned}\right.
$$

with $w>0$. Thus, on the average, a fraction $p$ of the $N(N-1) / 2$ couplings correspond to repulsive interaction, while the remaining couplings are attractive. The relative intensity of the repulsive interaction is $w$. Equations (11) can be rewritten as

$$
\dot{\phi}_{i}=\frac{1}{N} \sum_{j=1}^{N} \sin \left(\phi_{j}-\phi_{i}\right)-\frac{1+w}{N} \sum_{j \in \mathcal{R}_{i}} \sin \left(\phi_{j}-\phi_{i}\right) .
$$

In this representation, the first term in the r.h.s. of the equation corresponds to global attractive coupling, where all oscillator pairs interact with the same intensity. The second term corresponds to repulsive interaction of relative intensity $1+w$. Repulsive couplings are restricted to a random network determined by the sets $\mathcal{R}_{i}$. An oscillator $j$ belongs to $\mathcal{R}_{i}$ if it interacts repulsively with oscillator $i$. Calling $z_{i}$ the number of oscillators in $\mathcal{R}_{i}$, the average connectivity of the network of repulsive couplings is $\left\langle z_{i}\right\rangle=p(N-1)$.

Our aim here is to analyze the long-time solutions of Eqs. (3) as the parameters $p$ and $w$ are varied, averaging over different realizations of the network of repulsive couplings. Specifically, we are interested at the destabilization of full synchronization as the number and intensity of repulsive couplings grow. The state of full synchronization, $\phi_{i}(t)=\phi^{*}$ for all $i$ and constant $\phi^{*}$, is in fact a solution to Eqs. (3) for any $w$ and arbitrary $\mathcal{R}_{i}$. Its stability, however, is expected to depend on the intensity of the repulsive interaction and on the network topology. For $p=0$, which corresponds to the case of pure global attractive coupling, repulsive interaction is absent and full synchronization is stable. For $p=1$, coupling is also global but purely repulsive; the sets $\mathcal{R}_{i}$ are extended to the whole ensemble. In this situation, full synchronization is unstable and, for asymptotically large times, the system reaches one of the infinitely many states given by the equations $\sum_{j} \sin \left(\phi_{j}-\phi_{i}\right)=0(i=1, \ldots, N)$. In such states, typically, the phases $\phi_{i}$ are more or less uniformly distributed over $[0,2 \pi)[4]$. Destabilization of the fully synchronized state is thus expected to occur at intermediate values of $p$.

Linear stability of full synchronization can be assessed by studying the eigenvalue spectrum of the $N \times N$ matrix with elements

$$
S_{i j}=\frac{1}{N}+\left(-1+\frac{1+w}{N} z_{i}\right) \delta_{i j}-\frac{1+w}{N} R_{i j},
$$

where $\delta_{i j}$ are the elements of the identity matrix, and $R_{i j}$ are the elements of the (symmetric) adjacency matrix corresponding to the network of repulsive couplings: $R_{i j}=1$ if $j \in \mathcal{R}_{i}$, and 0 otherwise. The state of full synchronization is linearly stable if all the eigenvalues of $S_{i j}$ are negative.

Using a standard numerical routine, we have calculated the eigenvalues of $S_{i j}$ for different values of the parameters $p$ and $w$, and system sizes $N$ between 10 and $10^{3}$. For each parameter 


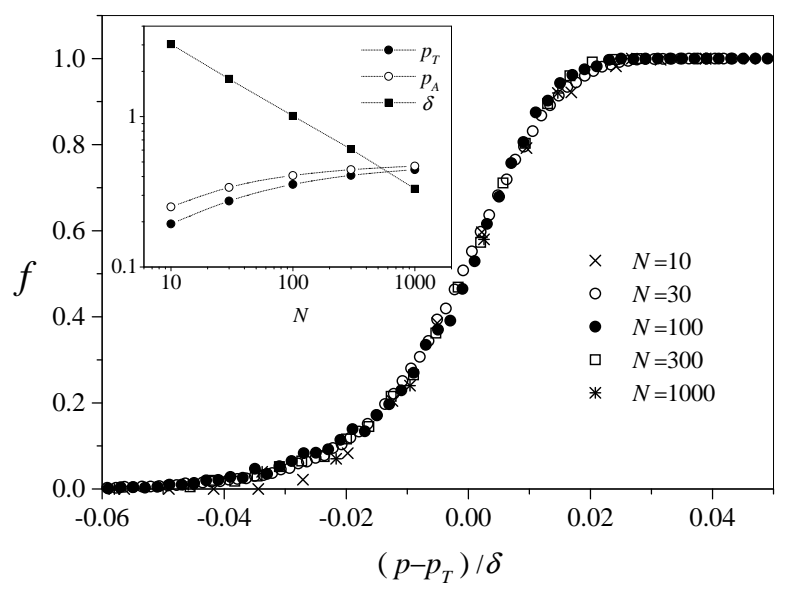

Fig. 1 - Data collapse of the fraction $f$ of realizations with unstable full synchronization, as a function of the fraction of repulsive couplings $p$, for different system sizes $N$. The intensity of the repulsive interaction is $w=1$. Inset: the transition point $p_{T}$ and the transition width $\delta$ as functions of $N$. The analytical result for the transition point, $p_{A}$, is also shown. Dotted lines are spline interpolations, added for clarity.

set, $10^{2}$ to $10^{4}$ realizations of the network of repulsive couplings have been considered. As a measure of the degree of instability of full synchronization, we have recorded the fraction $f$ of realizations for which at least one of the eigenvalues is positive -i.e., for which full synchronization is unstable. For fixed $N$ and $w$, as expected, this fraction changes from $f \approx 0$ for small $p$ to $f \approx 1$ for large $p$. The transition takes place in a rather narrow range of $p$. As illustrated in Fig. 团 for $w=1$, numerical results for $f$ as a function of $p$ and different system sizes admit a fairly good collapse as a function of the transformed parameter $\left(p-p_{T}\right) / \delta$. Here, $p_{T}$ has been chosen as the value of $p$ for which $f=1 / 2$, which we identify as the transition point. The coefficient $\delta$, in turn, measures the width of the transition range. For each size $N$, this coefficient is adjusted to achieve the data collapse, taking as a reference -without generality loss- $\delta=1$ for $N=100$. The inset of Fig. 1 shows a plot of $p_{T}$ and $\delta$ as functions of $N$. As $N$ grows, the transition point seems to approach an asymptotic value $p_{T} \approx 0.5$. The width $\delta$, on the other hand, decreases steadily, approximately following a power law $N^{-0.48 \pm 0.01}$. This indicates that the transition range becomes narrower, and the transition itself becomes better defined, as the system size is increased.

An independent, analytical evaluation of the transition point can be achieved under the hypothesis that the network of repulsive couplings is regular, i.e. when all the sets $\mathcal{R}_{i}$ have exactly the same number of elements, $z_{i}=z=p(N-1)$ for all $i$. In these conditions, the eigenvalues of $S_{i j}$ in Eq. (44) are directly related to the eigenvalues $\rho_{k}(k=1, \ldots, N)$ of $R_{i j}$. Consequently, the stability condition for full synchronization can be given in terms of $\rho_{k}$. The transition point turns out to be determined by the minimum eigenvalue of $R_{i j}$, $\rho_{\min }=\min \left\{\rho_{k}\right\}$. For sufficiently large $N$, the typical value of this eigenvalue can be estimated from the so-called semicircle law [8] which, in the present framework, establishes that the spectral density of $R_{i j}$ has the shape of a semicircle of radius $2 \sqrt{N p(1-p)}$ centered at the origin. The instability threshold for full synchronization is given by the condition [9]

$$
p\left(1-\frac{1}{N}\right)+2 \sqrt{\frac{p(1-p)}{N}}=\frac{1}{1+w} .
$$




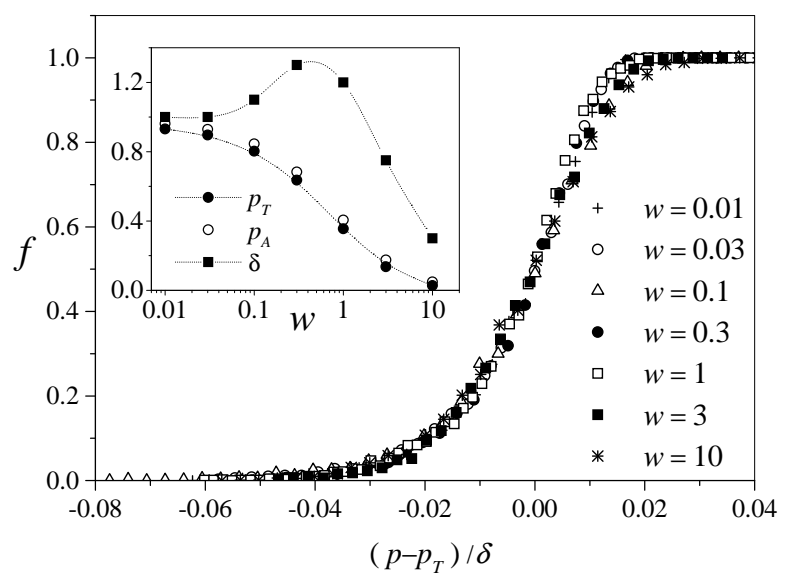

Fig. 2 - Data collapse of the fraction $f$ of realizations with unstable full synchronization, as a function of the fraction of repulsive couplings $p$, for different intensities $w$. The system size is $N=100$. Inset: the numerical and the analytical transition points, $p_{T}$ and $p_{A}$, and the transition width $\delta$, as functions of $w$. Dotted lines are spline interpolations, added for clarity.

The inset of Fig. 1 1 shows that the value $p_{A}$ obtained from this equation for $w=1$, as a function of the system size, is in very good qualitative agreement with $p_{T}$. Note that, for $N \rightarrow \infty$, our analytical evaluation predicts a transition point at $p_{A}=(1+w)^{-1}$; for $w=1$, we get $p_{A}=1 / 2$. Evaluation of the spectral density of $R_{i j}$ beyond the semicircle law $[10,11]$ shows that the fraction of eigenvalues outside the interval $(-2 \sqrt{N p(1-p)}, 2 \sqrt{N p(1-p)})$ decreases with the system size as $N^{-1}$. This is consistent with our observation that the transition becomes sharper as $N$ grows.

Figure 2 shows numerical results for $f$ in systems of fixed size, $N=100$, for various values of $w$. As in Fig. 1 the data collapse was achieved by plotting $f$ as a function of $\left(p-p_{T}\right) / \delta$, where $p_{T}$ is the fraction of repulsive couplings at which $f=1 / 2$. The inset shows $p_{T}$ and $\delta$ as functions of $w$. Since for both large and small $w$ the transition range is narrower than in between, $\delta$ reaches a maximum for an intermediate value of the intensity of the repulsive interaction. The analytical threshold $p_{A}$, obtained from Eq. (5), is again in very good qualitative agreement with the numerical results.

What is the nature of the stationary state of the oscillator ensemble just beyond the destabilization of full synchronization? To answer this question, we have inspected the longtime distribution of phases for networks of $N=100$ oscillators, where repulsive couplings were successively added. This has made it possible to detect, for each network, the exact point at which full synchronization becomes unstable, and to analyze the resulting unsynchronized asymptotic state. It turns out that this unsynchronized state changes qualitatively when the intensity of the repulsive interaction grows. For small and moderate $w$, destabilization of full synchronization gives place to a state where oscillator phases are irregularly spread over a small interval. Typically, the oscillator with maximal number of repulsive couplings has a substantially different phase. As expected, the distribution of phases widens as the fraction of repulsive couplings $p$ increases. These features are illustrated by the upper panels of Fig. 3. for $w=1$. In this particular realization of the oscillator network, full synchronization becomes unstable for $p=0.3527 \ldots$ The figure shows snapshots of the phase distribution in the plane $(\cos \phi, \sin \phi)$. Lines join oscillator pairs with repulsive couplings. 


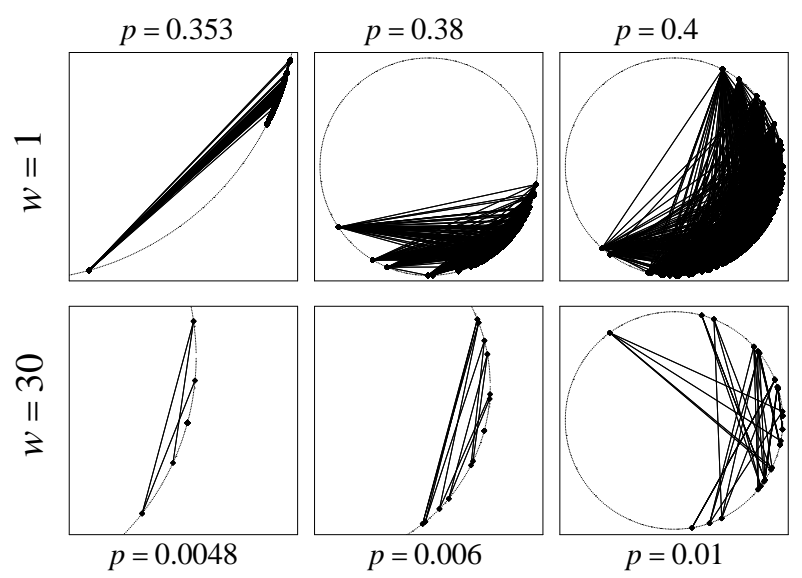

Fig. 3 - Long-time snapshots of the distribution of oscillator phases for different values of the intensity $w$ and fraction $p$ of repulsive couplings, in a system of size $N=100$. Individual phases are represented by dots on the unit circle (dotted line). Straight lines join oscillators which interact repulsively.

The situation changes substantially if the intensity of the repulsive interaction grows enough. For large $w$, when relatively few repulsive couplings are needed to destabilize full synchronization, unsynchronized states just beyond the transition are characterized by a symmetric distribution of phases. Some of the oscillator pairs with repulsive interaction abandon the synchronized cluster and their two elements reach symmetric positions at each side of the cluster. The remaining oscillators stay synchronized at a common phase. As the fraction of repulsive couplings keeps growing, the number of unsynchronized pairs increases, still preserving their symmetric configuration. Eventually, however, symmetry is broken, and the ensemble reaches a more or less irregular phase distribution, similar to that observed for lower $w$. The second line of panels in Fig. 3 illustrates these features for $w=30$. In this realization of the network, the transition takes place at $p=0.0048$, corresponding to just 24 repulsive couplings. For that value of $p$ and for $p=0.006$ we find symmetric configurations of phases, while for $p=0.01$ the distribution is already asymmetric. In the symmetric cases, the cluster of remaining synchronized oscillators is the isolated dot in the centre of the configuration.

Our numerical findings on the destabilization of full synchronization and the resulting unsynchronized states can be understood, at least qualitatively, by resorting to a concept borrowed from the study of disordered spin systems, namely, frustration $[6,12]$. In spin systems, where interactions are usually local, frustration is associated with the impossibility that three mutually interacting spins with interaction weights of different sign achieve simultaneously a state of minimal energy. In our system, where (attractive or repulsive) interaction affects every oscillator pair, frustration must be defined for each pair in relation to the state of the whole ensemble. For instance, an oscillator pair with repulsive coupling is frustrated if the system is in a collective synchronized state, so that the individual phases of the two oscillators are forced to coincide. Conversely, a pair with attractive coupling is frustrated if the interactions with the rest of the ensemble inhibits the synchronization of the two oscillators. Frustration is quantitatively characterized by the function

$$
F=-\frac{1}{N} \sum_{i, j=1}^{N} W_{i j} \cos \left(\phi_{j}-\phi_{i}\right) .
$$


Pairs with attractive coupling, $W_{i j}>0$, contribute to decrease the frustration $F$ if $\phi_{j} \approx \phi_{i}$. Vice versa, for $W_{i j}<0, F$ decreases as the difference of the two phases grows. The relevance of the frustration $F$ with respect to the oscillator dynamics resides in the fact that it plays the role of a nonequilibrium potential. Specifically, for $\omega=0$, Eqs. (11) can be written as $\dot{\phi}_{i}=-\partial F / \partial \phi_{i}$. Therefore,

$$
\dot{F}=\sum_{i=1}^{n} \frac{\partial F}{\partial \phi_{i}} \dot{\phi}_{i}=-\sum_{i=1}^{n}\left(\frac{\partial F}{\partial \phi_{i}}\right)^{2} \leq 0 .
$$

In other words, the dynamics convey a steady decrease of the frustration.

Bearing these considerations in mind, we can argue as follows. As the fraction of repulsive couplings grows from $p=0$, full synchronization is initially stable. Frustration, nevertheless, increases because of the increasing number of oscillators with repulsive interactions that are forced to stay into the synchronized cluster. The collective state of the ensemble is thus able to bear a certain degree of frustration, in order to maintain synchronization. Eventually, however, frustration reaches too high levels, and synchronization is no longer a "convenient" collective configuration -though it still is a possible stationary state for the ensemble. Consequently, the synchronized cluster breaks down, the individual oscillator phases spread out, and the level of frustration is alleviated. Since, now, oscillators are not locked to the synchronized cluster, further increase of $p$ will lead to the gradual widening of the distribution of phases, in the search for the state of minimal frustration.

When the repulsive interaction is strong enough, just a few repulsive couplings suffice to make full synchronization "inconvenient" from the viewpoint of frustration, and the synchronized cluster may break down for very small values of $p$. Under these conditions, the network of repulsive couplings is very simple: it typically consists of isolated oscillator pairs. If full synchronization is unstable, the two oscillators of each pair abandon the synchronized cluster, trying to maximize their mutual distance. At the same time, both of them tend to minimize the distance to the cluster, since their interaction with the oscillators there is attractive. The resulting configuration is thus symmetric around the cluster, as illustrated in Fig. 3 for $w=30$.

In summary, we have studied the desynchronization transition in a network of coupled identical phase oscillators with attractive and repulsive interactions, as the number and intensity of repulsive couplings is increased. For finite system sizes, the transition is gradual: the probability that the state of full synchronization is unstable for a given realization of the network of couplings grows smoothly with the number of repulsive couplings. Numerical finite-size analysis as well as analytical results, however, suggest that the desynchronization transition would become abrupt for infinitely large systems. Beyond the transition, the nature of the unsynchronized long-time state depends sensibly on the intensity of the repulsive interaction. For small and moderate intensities, individual oscillator phases reach a more or less disordered configuration, reminiscent of the disordered equilibrium state of an oscillator ensemble with global repulsive coupling [4]. On the other hand, for strong repulsive interaction, desynchronization gives rise to highly symmetric configurations, where the phase of unsynchronized oscillators are situated at both sides of the remaining synchronized cluster. The possibility of observing these symmetric configuration depends on their existence and stability, which we analyze in detail elsewhere [9]. The notion of frustration -already addressed, in the frame of coupled oscillator ensembles, by Daido [6]- is useful to construct a variational-like formulation for the dynamics of the system studied here. In this framework, we have given a qualitative description of our main results. Such formulation, however, should also play a role in a quantitatively detailed analysis of the system. In fact, as defined in Eq. (6) and 
under appropriate conditions at its singular points, frustration could be used as a Lyapunov function for the dynamics.

\section{REFERENCES}

[1] Winfree A. T., The Geometry of Biological Time (Springer, New York) 2001.

[2] Kuramoto Y., Chemical Oscillations, Waves, and Turbulence (Springer, Berlin) 1984.

[3] Pikovsky A., Rosemblum M. and Kurths J., Synchronization. A Universal Concept in Nonlinear Sciences. (Cambridge University Press, Cambridge) 2001, and references therein.

[4] Manrubia S. C., Mikhailov A. S. and Zanette D. H., Emergence of Dynamical Order. Synchronization Phenomena in Complex Systems (World Scientific, Singapore) 2004, and references therein.

[5] Daido H., Prog. Theor. Phys., 77 (1987) 622.

[6] Daido H., Phys. Rev. Lett., 68 (1992) 1073.

[7] Daido H., Phys. Rev. E, 61 (2000) 2145.

[8] Menta M. L.,Random Matrices (Academic Press, New York) 1991, 2nd edition.

[9] Zanette D. H., in preparation.

[10] Bronk B. V., J. Math. Phys., 5 (1964) 215.

[11] Farkas I. J., Derényi I., Barabási A.-L. and Vicsek T., Phys. Rev. E, 64 (2001) 026704.

[12] Mézard M., Parisi G. and Virasoro M. A., Spin Glass Theory and Beyond (World Scientific, Singapore) 1987. 